Published in final edited form as:

Pediatrics. 2013 November ; 132(5): 796-804. doi:10.1542/peds.2013-1493.

\title{
Influenza-Associated Pediatric Deaths in the United States, 2004-2012
}

\author{
Karen K. Wong, MD, MPH ${ }^{a}$, Seema Jain, MD $^{b}$, Lenee Blanton, MPH $^{b}$, Rosaline Dhara, MPH $^{b}$, \\ Lynnette Brammer, MPH ${ }^{\mathrm{b}}$, Alicia M. Fry, MD, MPH ${ }^{\mathrm{b}}$, and Lyn Finelli, DrPH ${ }^{\mathrm{b}}$ \\ aEpidemic Intelligence Service assigned to Influenza Division, Atlanta, Georgia \\ bInfluenza Division, Centers for Disease Control and Prevention, Atlanta, Georgia
}

\begin{abstract}
BACKGROUND-Influenza-associated deaths in children occur annually. We describe the epidemiology of influenza-associated pediatric deaths from the 2004-2005 through the 2011-2012 influenza seasons.

METHODS-Deaths in children $<18$ years of age with laboratory-confirmed influenza virus infection were reported to the Centers for Disease Control and Prevention by using a standard case report form to collect data on demographic characteristics, medical conditions, clinical course, and laboratory results. Characteristics of children with no high-risk medical conditions were compared with those of children with high-risk medical conditions.
\end{abstract}

\begin{abstract}
RESULTS-From October 2004 through September 2012, 830 pediatric influenza-associated deaths were reported. The median age was 7 years (interquartile range: 1-12 years). Thirty-five percent of children died before hospital admission. Of 794 children with a known medical history, $43 \%$ had no high-risk medical conditions, $33 \%$ had neurologic disorders, and $12 \%$ had genetic or chromosomal disorders. Children without high-risk medical conditions were more likely to die before hospital admission (relative risk: 1.9; 95\% confidence interval: 1.6-2.4) and within 3 days of symptom onset (relative risk: 1.6; 95\% confidence interval: 1.3-2.0) than those with high-risk medical conditions.
\end{abstract}

CONCLUSIONS-Influenza can be fatal in children with and without high-risk medical conditions. These findings highlight the importance of recommendations that all children should receive annual influenza vaccination to prevent influenza, and children who are hospitalized, who

\footnotetext{
Address correspondence to Karen K. Wong, MD, MPH, 1600 Clifton Rd NE, MS E-03, Atlanta, GA 30329. vij4@cdc.gov. The findings and conclusions in this report are those of the authors and do not necessarily represent the official position of the Centers for Disease Control and Prevention.

FINANCIAL DISCLOSURE: The authors have indicated they have no financial relationships relevant to this article to disclose. POTENTIAL CONFLICT OF INTEREST: The authors have indicated they have no potential conflicts of interest to disclose. Dr Wong conceptualized and designed the study, performed the statistical analysis, interpreted the data, and drafted the initial manuscript; Drs Jain and Fry conceptualized and designed the study, interpreted the data, and reviewed and revised the manuscript; Ms Blanton acquired, analyzed, and interpreted the data and reviewed and revised the manuscript; Ms Dhara acquired the data and reviewed and revised the manuscript; Ms Brammer acquired and interpreted the data and reviewed and revised the manuscript; Dr Finelli conceptualized and designed the study, supervised data collection, interpreted the data, and reviewed and revised the manuscript; and all authors approved the final manuscript as submitted.
} 
have severe illness, or who are at high risk of complications (age $<2$ years or with medical conditions) should receive antiviral treatment as early as possible.

\section{Keywords}

influenza; mortality; pediatric; surveillance

Influenza is associated with an estimated 54000 to 430000 hospitalizations and 3000 to 49 000 deaths annually, ${ }^{1-3}$ and influenza infection rates in the community are highest among children. ${ }^{4-6}$ Influenza is usually a self-limited illness, but severe complications, including pneumonia, encephalitis, myocarditis, and death, can occur in children. ${ }^{7-9}$

Since October 2004, influenza-associated pediatric deaths have been a nationally notifiable condition in the National Notifiable Diseases Surveillance System. This system has enabled characterization of severe influenza-associated disease, identification of at-risk groups for targeting prevention and treatment strategies, an devaluation of trends in influenzaassociated pediatric mortality over time..$^{7,8,10-15}$ These data have contributed to changes in the Advisory Committee on Immunization Practices (ACIP) recommendations for influenza vaccination. ${ }^{16-21}$ Previous studies of influenza-associated pediatrics deaths have described deaths over 1 or a few seasons, ${ }^{7,8,13,15}$ pandemic influenza A (H1N1) pdm09 (pH1N1) deaths, ${ }^{12,14}$ or deaths among children with certain risk factors. ${ }^{22}$ We describe influenzaassociated pediatric deaths reported since the national influenza-associated pediatric mortality surveillance system began in 2004 and compare characteristics of children with and without high-risk medical conditions who died.

\section{METHODS}

\section{Influenza-Associated Pediatric Death Surveillance}

An influenza-associated pediatric death is defined as a death in a US resident $<18$ years of age with laboratory-confirmed influenza virus infection. The confirmation of influenza virus infection can occur before or after death by any of the following laboratory methods: rapid diagnostic test, viral culture, fluorescent antibody, enzyme immunoassay, reverse transcription polymerase chain reaction, or immunohistochemical staining of tissue. Diagnostic testing is performed according to routine clinical care and/or postmortem examination if available. State and local health departments report influenza-associated pediatric deaths to the Centers for Disease Control and Prevention by using a standardized case report form that includes information about demographic characteristics, laboratory results, clinical course, medical conditions, and influenza vaccination status. Health departments verify vaccination status by reviewing medical records, contacting the child's health care provider, or checking the state vaccine registry, if available. Bacterial coinfection is defined as bacteria cultured from a normally sterile site (blood, cerebrospinal fluid, pleural fluid) after illness onset or from postmortem lung tissue if the specimen was collected $<24$ hours after death.

An influenza season was defined as October 1 through September 30 with the exception of the $\mathrm{pH} 1 \mathrm{~N} 1$ period, during which seasons were defined as October 1, 2008, through August 
29, 2009, and August 30, 2009, through September 30, 2010. Rates of influenza-associated death were reported with respect to census estimates of the population $<18$ years of age during the same influenza season. ${ }^{23}$ The ACIP high-risk medical conditions include the following: asthma, neurologic/neurodevelopmental disorders, chronic lung disease, heart disease including congenital heart disease, blood disorders, endocrine disorders, kidney disorders, liver disorders, metabolic disorders, immunosuppression, and pregnancy. ${ }^{20}$ In this analysis, children with neurologic/neurodevelopmental disorders were considered to have a high-risk medical condition for all influenza seasons. Analyses of influenza antiviral treatment data were limited to seasons in which they were consistently collected (2010-2011 and 2011-2012).

Groups recommended for influenza vaccination by the ACIP changed during the study period. During the 2004-2005 and 2005-2006 seasons, all children 6 to 23 months of age and children $<18$ years of age with high-risk medical conditions were recommended for vaccination. ${ }^{16,17}$ Neurologic disorders were added to the ACIP high-risk medical conditions in 2005. ${ }^{17}$ In the 2006-2007 and 2007-2008 seasons, vaccination recommendations were expanded to all children 6 to 59 months of age. ${ }^{18}$ This recommendation was expanded further to all children 6 months to 18 years of age in the 2008-2009 season. ${ }^{19-21}$ All children $\succ 6$ months of age were considered eligible for influenza vaccination in this analysis, even if they were not specifically recommended for vaccination by the ACIP on the basis of age, medical conditions, or household contacts during the season of their death, because vaccine formulations approved by the Food and Drug Administration for persons $\geq 6$ months of age were available during the entire study period. During the 2009-2010 season, the group eligible for the $\mathrm{pH} 1 \mathrm{~N} 1$ monovalent vaccine was limited in this analysis to those who died on or after November 1, 2009, to account for timing of vaccine distribution. Children were considered fully vaccinated if they received the recommended number of doses of influenza vaccine $\geq 14$ days before illness onset that season. Selected characteristics were compared between children with and without high-risk medical conditions.

\section{Analysis}

Data were analyzed by using SAS, version 9.3 (SAS Institute, Cary, NC). A Wilcoxon ranksum test was used to evaluate differences between non-normally distributed data. A $t$ test was used to evaluate differences between means, and a $\chi^{2}$ test was used to evaluate differences between proportions. Asymptotic 95\% confidence intervals (CIs) are reported for relative risks (RRs) where the natural $\log$ of the RR is assumed to be approximately normally distributed. All $P$ values are 2 -sided, and $P<.05$ was considered significant.

\section{RESULTS}

\section{Characteristics of Influenza-Associated Deaths in Children}

As of August 2, 2013, a total of 830 laboratory-confirmed influenza-associated pediatric deaths occurring from October 1, 2004, through September 30, 2012, were reported from all 50 states and Guam. The most deaths reported in 1 season, 282 (3.8 deaths per 1 million children), occurred during the 2009-2010 season, and the fewest deaths, 35 ( 0.5 deaths per 1 million children), occurred during the 2011-2012 season (Fig 1). Deaths for most seasons 
peaked in February or March (Fig 2). After the pH1N1 virus was identified in the United States in April 2009, an early wave of pediatric deaths occurred during June 2009 followed by a larger peak in October 2009.

Of children who died, 53\% were male (Table 1). The median age of children was 7 years. Children who died during the 2005-2006 season had the youngest median age of 3 years, and children who died during the 2009-2010 season had the oldest median age of 8.5 years (Table 2). Overall, 649 (78\%) children with influenza-associated death had influenza A virus infection, $165(20 \%)$ had influenza B virus infection, and $1(<1 \%)$ was coinfected with influenza A and B viruses; 15 (2\%) children had influenza virus infection for which the type was not distinguished. Except for the 2009-2010 season during which only $1 \%$ of viruses identified were influenza B, influenza B virus infection was identified in $23 \%$ to $38 \%$ of deaths each season (Table 2).

Of 794 children with influenza-associated death and a reported medical history, 453 (57\%) had $\geq 1$ high-risk medical condition. Neurologic disorders were the most commonly reported high-risk medical condition (33\%). Overall, $26 \%$ had a pulmonary disorder, including $16 \%$ with asthma; $12 \%$ had a chromosome or genetic abnormality associated with a high-risk condition; and $11 \%$ had congenital heart disease or other cardiac disease (Table 1).

Approximately one-third or more of children who died each season had no known high-risk medical conditions, and during the 2006-2007 season the majority (62\%) of children had no high-risk medical conditions (Table 2).

Among children with influenza-associated death, the proportion without high-risk medical conditions varied by the type and subtype of influenza virus identified $(P<.001)$. Among 323 deaths associated with pH1N1 virus, 115 (36\%) occurred in children with no high-risk medical conditions. For deaths associated with seasonal influenza A (H1N1), influenza A (H3N2), and influenza B viruses, the proportions occurring in children without high-risk medical conditions were 20 of 32 (63\%), 41 of 57 (72\%), and 72 of $150(48 \%)$ children, respectively.

More than one-third of children died before hospital admission, including 18\% who died in the emergency department and 16\% who died outside the hospital (Table 1). The median duration of illness was 5 days (interquartile range: 3-12 days). Of children who had a bacterial culture obtained from a normally sterile site, 154 (40\%) had $\geq 1$ bacterial coinfection. Almost half of those with bacterial coinfections had Staphylococcus aureus infection, and $22(14 \%)$ had Streptococcus pneumoniae infection. Other bacterial coinfections identified included Streptococcus species other than $S$ pneumoniae $(n=38)$, of which the most common was Streptococcus pyogenes $(n=20)$, Enterococcus species ( $n=$ 5), and Gram-negative organisms such as Pseudomonas aeruginosa $(n=6)$, Enterobacter cloacae $(n=4)$, and Klebsiella pneumoniae $(n=3)$. Among 769 children with information on complications, the most common complication was radiographically confirmed pneumonia (51\%); other complications included seizures (11\%), encephalopathy/ encephalitis (9\%), and coinfection with noninfluenza viruses (5\%) (Table 1). 
Among 126 children who died during the 2010-2011 and 2011-2012 seasons whose treatment status was known, 56 (44\%) received influenza antiviral treatment. When limiting those deaths to the 74 that occurred after hospital admission, 46 (62\%) received antiviral treatment; and when limiting further to the 65 children $\geq 1$ year of age, $43(66 \%)$ received antiviral treatment. Of 511 children $\ 6$ months of age whose vaccination status was known, $84(16 \%)$ had been fully vaccinated with seasonal influenza vaccine. During the 2009-2010 season, of 66 children $\nsucceq 6$ months of age whose $\mathrm{pH} 1 \mathrm{~N} 1$ vaccination status was known and who died on or after November 1, 2009, $2(3 \%)$ had been fully vaccinated with pH1N1 monovalent vaccine.

\section{Comparison of Influenza-Associated Deaths in Children Without and With High-Risk Medical Conditions}

There were 341 (43\%) children without high-risk medical conditions and 453 (57\%)

children with $\geq 1$ high-risk medical condition who died; medical history was unknown for 36 children. Children without high-risk medical conditions were more likely to be $<5$ years old than those with high-risk medical conditions (RR: 1.3; 95\% CI: 1.1-1.6) (Table 3). Those without high-risk medical conditions were more likely to die outside the hospital or in the emergency department (RR: 1.9; 95\% CI: 1.6-2.4) and $₫$ days after illness onset (RR: 1.6; 95\% CI: 1.3-2.0). Among children who were tested, bacterial coinfection was more common among children without high-risk medical conditions (RR: 2.0; 95\% CI: 1.5-2.5).

Among those who $\ 6$ months of age whose vaccination status was known, 19 of 203 (9\%) children without high-risk medical conditions and 64 of 290 (22\%) children with high-risk medical conditions had been fully vaccinated with seasonal influenza vaccine. During the 2010-2011 and 2011-2012 seasons, children without high-risk medical conditions were less likely to receive influenza antiviral treatment than those with high-risk medical conditions, although the difference was not statistically significant (35\% vs 54\%; RR: 0.7 ; $95 \%$ CI: $0.4-$ $1.0)$.

\section{DISCUSSION}

This study describes 830 deaths among children reported to the national influenza-associated pediatric mortality surveillance system from the 2004-2005 through the 2011-2012 influenza seasons and reveals the importance of current strategies for the prevention and treatment of influenza in children. Deaths occurred in children of all ages and regardless of high-risk medical conditions. However, children with neurologic disorders, chromosome abnormalities, and genetic disorders were highly represented among those who died compared with the prevalence of these disorders in the general pediatric population. Influenza-associated pediatric deaths often occurred within days of symptom onset. Bacterial coinfections and pneumonia were commonly reported among children who died. Efforts to prevent influenza and its complications in children by ensuring annual influenza vaccination and appropriate early access to antiviral medications should remain a priority for the medical and public health communities.

Children with high-risk medical conditions are vulnerable to severe influenza-associated complications, including death. ${ }^{24}$ These conditions include common childhood conditions, 
such as asthma. Some conditions, such as neurologic disorders and conditions associated with underlying chromosome and genetic abnormalities, were over-represented in our data compared with the prevalence of these disorders in the general pediatric population. Cerebral palsy, which affects $<1 \%$ of children in the United States, ${ }^{25}$ was reported among $10 \%$ of children with influenza-associated death in this analysis. The high prevalence of cerebral palsy among the fatalities is consistent with studies highlighting the vulnerability of these children to poor outcomes after influenza virus infection. ${ }^{22,24,26}$ It is especially important that children with neurologic disorders and other high-risk conditions, who often have more regular access to health care than otherwise healthy children, ${ }^{27}$ receive influenza vaccination before the influenza season begins and early antiviral treatment of suspected influenza. ${ }^{21,28}$ Clinicians should maintain a high index of suspicion for influenza virus infection in children with high-risk conditions when influenza is circulating in their communities.

Previously healthy children of all ages are also at risk of influenza-associated death. In this study, $>40 \%$ of children who died had no high-risk medical conditions. Among children hospitalized with laboratory-confirmed influenza in the United States, the percentage with no high-risk medical conditions ranged from $47 \%$ to $59 \%$ over the same period as this study. ${ }^{29}$ Healthy children are often perceived as not being at risk of serious influenza illness. A 2003 survey of parents of healthy children found that more than half believed that influenza infections are more serious in healthy 70 -year-olds than in healthy 1 -year-olds ${ }^{30}$; however, the risk of influenza-associated hospitalization in young children is similar to that of older adults. ${ }^{2,31}$ Parents and clinicians should be aware that influenza can be associated with severe complications in otherwise healthy children, especially in those who are $<2$ years of age. ${ }^{13}$

Influenza-associated deaths often occurred quickly, and some ill children were not admitted to a hospital before they died. More than one-third of children died outside the hospital or in the emergency department, and most children died within 1 week of symptom onset. Previously healthy children appeared to have a shorter interval between symptom onset and death compared with children with high-risk medical conditions. The reasons for this observation are unknown, and the clinical course of influenza in these 2 populations has not been directly compared previously. Immune dysregulation may lead to severe illness in some previously healthy children. ${ }^{32}$ In this study, previously healthy children were more likely than those with high-risk medical conditions to have a bacterial coinfection, which may contribute to the difference in clinical course observed between the 2 groups. The potential for rapid clinical decline in children with influenza virus infection emphasizes the importance of reducing the risk of influenza infection and related complications through prevention and early treatment.

Pneumonia was the most frequently reported complication among children who died. Children hospitalized with influenza-associated pneumonia have more severe clinical outcomes, including respiratory failure and death, than children hospitalized with influenza without pneumonia. ${ }^{33,34}$ Bacterial coinfections were also a complication identified in many children who died, with $S$ aureus and Streptococcus species the pathogens most commonly identified. Severe outcomes among children with influenza and bacterial coinfection, including coagulopathy, ICU admission, and death, have been described. ${ }^{34-37}$ Empirical 
antibiotic and influenza antiviral medication is recommended for treatment of children with community-acquired pneumonia and suspected influenza virus infection. ${ }^{28,38}$ Children $<5$ years of age are recommended to receive pneumococcal conjugate vaccine, and children $\geq 2$ years of age who are at high risk of pneumococcal disease should also receive the pneumococcal polysaccharide vaccine. ${ }^{39}$

Influenza vaccination is the best prevention against influenza virus infection and its complications, and these data, which reveal that any child can be at risk of influenzaassociated death regardless of age or high-risk medical conditions, support the recommendation that all children $\succ 6$ months of age receive annual influenza vaccination. ${ }^{21}$ Few children who died were vaccinated. National influenza vaccine coverage among children 6 months to 17 years of age increased ${ }^{40,41}$ from $24 \%$ in $2008-2009$ to $52 \%$ in 2011-2012 but remained well below the Healthy People 2020 target of $80 \% .{ }^{42}$ Infants $<6$ months of age are at higher risk of influenza-related complications but are too young to be vaccinated; to protect them, pregnant women and close contacts of infants are recommended for vaccination. ${ }^{20,43,44}$ Children with high-risk medical conditions, who were overrepresented among the fatalities in this report, as well as their close contacts, are important target groups for influenza prevention strategies. Studies in adult nursing facility staff suggest that vaccinating contacts of high-risk persons can reduce influenza morbidity and mortality among residents at high risk. ${ }^{45-47}$

In addition to influenza vaccination, influenza antiviral medications can reduce the severity of illness and complications associated with influenza virus infection. ${ }^{28}$ Antiviral treatment can shorten illness duration in children with uncomplicated influenza, ${ }^{48-50}$ decrease the need for mechanical ventilation in children hospitalized with influenza, ${ }^{51}$ and lower the odds of death in critically ill children with pH1N1 virus infection ${ }^{52}$; however, antiviral treatment was reported in less than half of the children who died during the 2010-2011 and 20112012 seasons in this study. Previous studies have shown that antiviral treatment among children hospitalized with influenza increased sharply during the 2009 pandemic compared with prepandemic seasons, ${ }^{53}$ but it decreased from $77 \%$ during the pandemic to $56 \%$ during the 2010-2011 season, ${ }^{54}$ even though children hospitalized with suspected influenza are recommended to receive early empirical antiviral treatment. ${ }^{55}$ Children with signs or symptoms of severe or progressive illness and those who are hospitalized should be treated empirically for influenza with antiviral medications such as oseltamivir, which is approved by the Food and Drug Administration for treatment of influenza in persons $\geq 2$ weeks of age, or zanamivir without waiting for laboratory results, even if they have no other risk factors for influenza-associated complications. In addition, children $<2$ years of age or those with highrisk medical conditions are recommended to receive antiviral medication for treatment no matter the severity of illness. ${ }^{28,55}$

This report is subject to limitations. The national influenza-associated pediatric mortality surveillance system requires laboratory diagnosis of influenza virus infection and will miss deaths for which influenza testing is not available or routinely performed. Testing practices and patterns of health care use are likely to differ between children with and without highrisk medical conditions; those with high-risk medical conditions may be more likely to be tested for influenza and therefore more likely to be reported in the surveillance system. 
Influenza testing was more prevalent during the $\mathrm{pH} 1 \mathrm{~N} 1$ influenza pandemic, leading to increased case reporting during that time period. Information in the case report form is collected by state and local health officials and is not systematically verified against the medical record or autopsy report, which may lead to misclassification or underreporting of high-risk medical conditions, complications, vaccination status, and antiviral treatment. Finally, data on the timing and duration of influenza antiviral treatment were not collected, thus limiting the interpretation of antiviral treatment impact.

\section{CONCLUSIONS}

Although influenza usually causes a self-limited illness, influenza can also be associated with severe outcomes in children, including death. Deaths can occur within a few days after illness onset. The potential for severe outcomes from influenza should be recognized in all children, both those with conditions that place them at higher risk of influenza-associated complications as well as healthy children. The findings of this national surveillance system report highlight the importance of influenza vaccination for all children $\searrow$ months of age and early empirical antiviral treatment of all children with suspected or confirmed influenza virus infection who are hospitalized; who have severe, complicated, or progressive illness; or who are at higher risk of influenza-associated complications due to younger age ( $<2$ years) or medical conditions.

\section{Acknowledgments}

FUNDING: No external funding.

\section{ABBREVIATIONS}

ACIP Advisory Committee on Immunization Practices

CI confidence interval

pH1N1 influenza A (H1N1) pdm09

RR relative risk

\section{References}

1. Centers for Disease Control and Prevention. Estimates of deaths associated with seasonal influenza —United States, 1976-2007. MMWR Morb Mortal Wkly Rep. 2010; 59(33):1057-1062. [PubMed: 20798667]

2. Thompson WW, Shay DK, Weintraub E, et al. Influenza-associated hospitalizations in the United States. JAMA. 2004; 292(11):1333-1340. [PubMed: 15367555]

3. Thompson WW, Shay DK, Weintraub E, et al. Mortality associated with influenza and respiratory syncytial virus in the United States. JAMA. 2003; 289(2):179-186. [PubMed: 12517228]

4. Fox JP, Hall CE, Cooney MK, Foy HM. Influenzavirus infections in Seattle families, 1975-1979. I. Study design, methods and the occurrence of infections by time and age. Am J Epidemiol. 1982; 116(2):212-227. [PubMed: 7114033]

5. Monto AS, Kioumehr F. The Tecumseh Study of Respiratory Illness. IX. Occurence of influenza in the community, 1966-1971. Am J Epidemiol. 1975; 102(6):553-563. [PubMed: 1202957] 
6. Fowlkes A, Dasgupta S, Chao E, et al. Estimating influenza incidence and rates of influenza-like illness in the outpatient setting. Influenza Other Respi Viruses. 2013 Sep; 7(5):694-700.

7. Bhat N, Wright JG, Broder KR, et al. Influenza Special Investigations Team. Influenza-associated deaths among children in the United States, 2003-2004. N Engl J Med. 2005; 353(24):2559-2567. [PubMed: 16354892]

8. Finelli L, Fiore A, Dhara R, et al. Influenza-associated pediatric mortality in the United States: increase of Staphylococcus aureus coinfection. Pediatrics. 2008; 122(4):805-811. [PubMed: 18829805]

9. Paddock CD, Liu L, Denison AM, et al. Myocardial injury and bacterial pneumonia contribute to the pathogenesis of fatal influenza B virus infection. J Infect Dis. 2012; 205(6):895-905. [PubMed: 22291193]

10. Centers for Disease Control and Prevention. Update: influenza-associated deaths reported among children aged <18 years-United States, 2003-04 influenza season. MMWR Morb Mortal Wkly Rep. 2004; 52(51-52):1254-1255. [PubMed: 14704651]

11. Centers for Disease Control and Prevention. Update: influenza-associated deaths reported among children aged <18 years-United States, 2003-04 influenza season. MMWR Morb Mortal Wkly Rep. 2004; 52(53):1286-1288. [PubMed: 14712178]

12. Centers for Disease Control and Prevention. Surveillance for pediatric deaths associated with 2009 pandemic influenza A (H1N1) virus infection-United States, April-August 2009. MMWR Morb Mortal Wkly Rep. 2009; 58(34):941-947. [PubMed: 19730406]

13. Centers for Disease Control and Prevention. Influenza-associated pediatric deaths-United States, September 2010-August 2011. MMWR Morb Mortal Wkly Rep. 2011; 60(36):1233-1238. [PubMed: 21918492]

14. Cox CM, Blanton L, Dhara R, Brammer L, Finelli L. 2009 Pandemic influenza A (H1N1) deaths among children-United States, 2009-2010. Clin Infect Dis. 2011; 52(suppl 1):S69-S74. [PubMed: 21342902]

15. Peebles PJ, Dhara R, Brammer L, Fry AM, Finelli L. Influenza-associated mortality among children-United States: 2007-2008. Influenza Other Respi Viruses. 2011; 5(1):25-31.

16. Harper SA, Fukuda K, Uyeki TM, et al. Centers for Disease Control and Prevention. Prevention and control of influenza: recommendations of the Advisory Committee on Immunization Practices (ACIP). MMWR Recomm Rep. 2004 May 28; 53(RR-6):1-40.

17. Harper SA, Fukuda K, Uyeki TM, et al. Centers for Disease Control and Prevention (CDC). Prevention and control of influenza: recommendations of the Advisory Committee on Immunization Practices (ACIP). MMWR Recomm Rep. 2005 Jul 29; 54(RR-8):1-40.

18. Smith NM, Bresee JS, Shay DK, et al. Advisory Committee on Immunization Practices. Prevention and control of Influenza: recommendations of the Advisory Committee on Immunization Practices (ACIP). MMWR Recomm Rep. 2006; 55(RR-10):1-42.

19. Fiore AE, Shay DK, Broder K, et al. Centers for Disease Control and Prevention (CDC). Prevention and control of influenza: recommendations of the Advisory Committee on Immunization Practices (ACIP), 2008. MMWR Recomm Rep. 2008 Aug 8; 57(RR-7):1-60.

20. Fiore AE, Uyeki TM, Broder K, et al. Centers for Disease Control and Prevention. Prevention and control of influenza with vaccines: recommendations of the Advisory Committee on Immunization Practices (ACIP), 2010. MMWR Recomm Rep. 2010; 59(RR-8):1-62.

21. Centers for Disease Control and Prevention. Prevention and control of influenza with vaccines: recommendations of the Advisory Committee on Immunization Practices (ACIP), 2011. MMWR Morb Mortal Wkly Rep. 2011; 60(33):1128-1132. [PubMed: 21866086]

22. Blanton L, Peacock G, Cox C, Jhung M, Finelli L, Moore C. Neurologic disorders among pediatric deaths associated with the 2009 pandemic influenza. Pediatrics. 2012; 130(3):390-396. [PubMed: 22931899]

23. US Census Bureau. [Accessed July 19, 2013] Annual estimates of the resident population for selected age groups by sex for the United States, states, counties, and Puerto Rico commonwealth and municipios: April 1, 2010 to July 1, 2012. Published June 2013. Available at: http:// factfinder2.census.gov 
24. Coffin SE, Zaoutis TE, Rosenquist AB, et al. Incidence, complications, and risk factors for prolonged stay in children hospitalized with community-acquired influenza. Pediatrics. 2007; 119(4):740-748. [PubMed: 17403845]

25. Kirby RS, Wingate MS, Van Naarden Braun K, et al. Prevalence and functioning of children with cerebral palsy in four areas of the United States in 2006: a report from the Autism and Developmental Disabilities Monitoring Network. Res Dev Disabil. 2011; 32(2):462-469. [PubMed: 21273041]

26. Keren R, Zaoutis TE, Bridges CB, et al. Neurological and neuromuscular disease as a risk factor for respiratory failure in children hospitalized with influenza infection. JAMA. 2005; 294(17): 2188-2194. [PubMed: 16264160]

27. Silver EJ, Stein RE. Access to care, unmet health needs, and poverty status among children with and without chronic conditions. Ambul Pediatr. 2001; 1(6):314-320. [PubMed: 11888421]

28. Fiore AE, Fry A, Shay D, Gubareva L, Bresee JS, Uyeki TM. Centers for Disease Control and Prevention. Antiviral agents for the treatment and chemoprophylaxis of influenzarecommendations of the Advisory Committee on Immunization Practices (ACIP). MMWR Recomm Rep. 2011; 60(1):1-24.

29. Centers for Disease Control and Prevention. [Accessed March 15, 2013] FluView: influenza hospitalization surveillance network. Available at: http://gis.cdc.gov/GRASP/Fluview/ FluHospChars.html

30. Daley MF, Crane LA, Chandramouli V, et al. Misperceptions about influenza vaccination among parents of healthy young children. Clin Pediatr (Phila). 2007; 46(5):408-417. [PubMed: 17556737]

31. Neuzil KM, Mellen BG, Wright PF, Mitchel EF Jr, Griffin MR. The effect of influenza on hospitalizations, outpatient visits, and courses of antibiotics in children. N Engl J Med. 2000; 342(4):225-231. [PubMed: 10648763]

32. Heltzer ML, Coffin SE, Maurer K, et al. Immune dysregulation in severe influenza. J Leukoc Biol. 2009; 85(6):1036-1043. [PubMed: 19276177]

33. Dawood FS, Fiore A, Kamimoto L, et al. Emerging Infections Program (EIP) Network. Influenzaassociated pneumonia in children hospitalized with laboratory-confirmed influenza, 2003-2008. Pediatr Infect Dis J. 2010; 29(7):585-590. [PubMed: 20589966]

34. Spaeder MC, Milstone AM, Fackler JC. Association of bacterial pneumonia and respiratory failure in children with community-acquired influenza infection. Pediatr Crit Care Med. 2011; 12(4):e181-e183. [PubMed: 21037508]

35. Hernández-Bou S, Novell CB, Alins JG, García-García JJ. Infectious Diseases Working Group, Spanish Society of Pediatric Emergencies. Hospitalized children with influenza A H1N1 (2009) infection: a Spanish multicenter study. Pediatr Emerg Care. 2013; 29(1):49-52. [PubMed: 23283263]

36. Nguyen T, Kyle UG, Jaimon N, et al. Coinfection with Staphylococcus aureus increases risk of severe coagulopathy in critically ill children with influenza A (H1N1) virus infection. Crit Care Med. 2012; 40(12):3246-3250. [PubMed: 22971587]

37. Reed C, Kallen AJ, Patton M, et al. Infection with community-onset Staphylococcus aureus and influenza virus in hospitalized children. Pediatr Infect Dis J. 2009; 28(7):572-576. [PubMed: 19478685]

38. Bradley JS, Byington CL, Shah SS, et al. Pediatric Infectious Diseases Society; Infectious Diseases Society of America. The management of community-acquired pneumonia in infants and children older than 3 months of age: clinical practice guidelines by the Pediatric Infectious Diseases Society and the Infectious Diseases Society of America. Clin Infect Dis. 2011; 53(7):e25-e76. [PubMed: 21880587]

39. Nuorti JP, Whitney CG. Centers for Disease Control and Prevention. Prevention of pneumococcal disease among infants and children-use of 13-valent pneumococcal conjugate vaccine and 23valent pneumococcal polysaccharide vaccine: recommendations of the Advisory Committee on Immunization Practices (ACIP). MMWR Recomm Rep. 2010; 59(RR-11):1-18. 
40. Centers for Disease Control and Prevention. Influenza vaccination coverage among children and adults-United States, 2008-09 influenza season. MMWR Morb Mortal Wkly Rep. 2009; 58(39): 1091-1095. [PubMed: 19816396]

41. Centers for Disease Control and Prevention. [Accessed January 2, 2013] Flu vaccination coverage, United States, 2011-12 influenza season. Available at: www.cdc.gov/flu/pdf/professionals/ vaccination/vax-coverage-1112estimates.pdf

42. US Department of Health and Human Services, Office of Disease Prevention and Health Promotion. [Accessed January 2, 2013] Healthy People. 2020. Available at: www.healthypeople.gov/2020/topicsobjectives2020/pdfs/HP2020objectives.pdf

43. Steinhoff MC, Omer SB, Roy E, et al. Influenza immunization in pregnancy-antibody responses in mothers and infants. N Engl J Med. 2010; 362(17):1644-1646. [PubMed: 20427817]

44. Zaman K, Roy E, Arifeen SE, et al. Effectiveness of maternal influenza immunization in mothers and infants. N Engl J Med. 2008; 359(15):1555-1564. [PubMed: 18799552]

45. Hayward AC, Harling R, Wetten S, et al. Effectiveness of an influenza vaccine programme for care home staff to prevent death, morbidity, and health service use among residents: cluster randomised controlled trial. BMJ. 2006; 333:1241. [PubMed: 17142257]

46. Lemaitre M, Meret T, Rothan-Tondeur M, et al. Effect of influenza vaccination of nursing home staff on mortality of residents: a cluster-randomized trial. J Am Geriatr Soc. 2009; 57(9):15801586. [PubMed: 19682118]

47. Shugarman LR, Hales C, Setodji CM, Bardenheier B, Lynn J. The influence of staff and resident immunization rates on influenza-like illness outbreaks in nursing homes. J Am Med Dir Assoc. 2006; 7(9):562-567. [PubMed: 17095421]

48. Hedrick JA, Barzilai A, Behre U, et al. Zanamivir for treatment of symptomatic influenza A and B infection in children five to twelve years of age: a randomized controlled trial. Pediatr Infect Dis J. 2000; 19(5):410-417. [PubMed: 10819336]

49. Heinonen S, Silvennoinen H, Lehtinen $P$, et al. Early oseltamivir treatment of influenza in children 1-3 years of age: a randomized controlled trial. Clin Infect Dis. 2010; 51(8):887-894. [PubMed: 20815736]

50. Whitley RJ, Hayden FG, Reisinger KS, et al. Oral oseltamivir treatment of influenza in children. Pediatr Infect Dis J. 2001; 20(2):127-133. [PubMed: 11224828]

51. Eriksson CO, Graham DA, Uyeki TM, Randolph AG. Risk factors for mechanical ventilation in U.S. children hospitalized with seasonal influenza and 2009 pandemic influenza A. Pediatr Crit Care Med. 2012; 13(6):625-631. [PubMed: 22895006]

52. Farias JA, Fernández A, Monteverde E, et al. Critically ill infants and children with influenza A (H1N1) in pediatric intensive care units in Argentina. Intensive Care Med. 2010; 36(6):1015-1022. [PubMed: 20237757]

53. Hersh AL, Herigon JC, Ampofo K, Pavia AT, Newland JG. Rapid increase in use of antiviral therapy for hospitalized children with influenza during the 2009 H1N1 epidemic. Pediatr Infect Dis J. 2011; 30(10):895-897. [PubMed: 21587093]

54. Garg S, Chaves SS, Pérez A, et al. Reduced influenza antiviral treatment among children and adults hospitalized with laboratory-confirmed influenza infection in the year after the 2009 pandemic. Clin Infect Dis. 2012; 55(3):e18-e21. [PubMed: 22543024]

55. Committee on Infectious Diseases, American Academy of Pediatrics. Recommendations for prevention and control of influenza in children, 2012-2013. Pediatrics. 2012; 130(4):780-792. [PubMed: 22966032] 


\section{WHAT'S KNOWN ON THIS SUBJECT}

Influenza-associated deaths in children occur every year among children of all ages. Young children and those with high-risk medical conditions are at higher risk of influenza-related complications.

\section{WHAT THIS STUDY ADDS}

This study describes influenza-associated pediatric deaths over 8 influenza seasons in the United States and compares characteristics of deaths in children with high-risk medical conditions with those in children without high-risk medical conditions. 


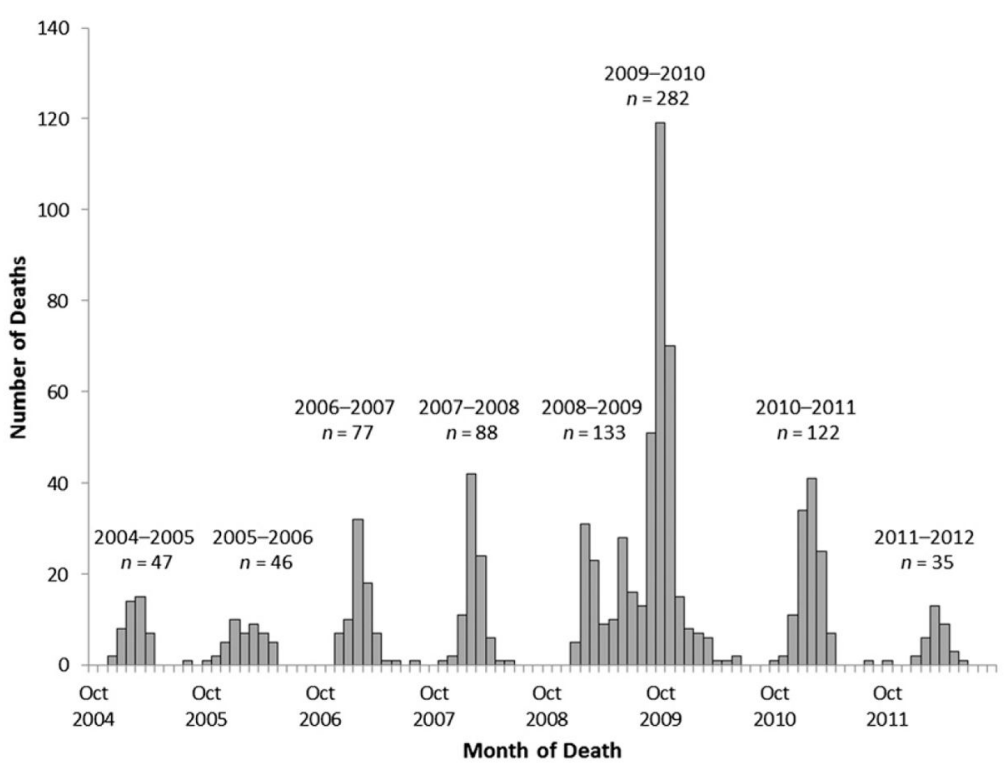

FIGURE 1.

Number of influenza-associated pediatric deaths by month of death $(N=830)$ : United States, October 2004 through September 2012. 


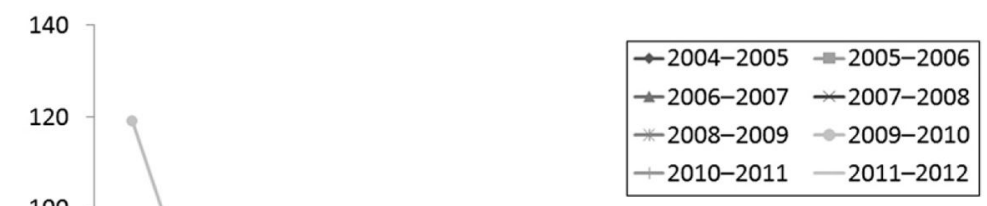

\section{FIGURE 2 .}

Influenza-associated pediatric deaths $(N=830)$ by month of death and influenza season: United States, October 2004 through September 2012. 
TABLE 1

Selected Characteristics Among Children With Influenza-Associated Death: United States, October 2004 Through September 2012

\begin{tabular}{|c|c|}
\hline Characteristic & Value \\
\hline Male gender & $442(53)$ \\
\hline Age, median (IQR), y & $7(1-12)$ \\
\hline$<6$ months & $94(11)$ \\
\hline 6 to 23 months & $114(14)$ \\
\hline 24 to 59 months & $117(14)$ \\
\hline 5 to 8 years & $167(20)$ \\
\hline 9 to 12 years & $112(13)$ \\
\hline 13 to 17 years & $226(27)$ \\
\hline \multicolumn{2}{|l|}{ Race/ethnicity ${ }^{a}$} \\
\hline White & $373(45)$ \\
\hline Hispanic or Latino & $194(23)$ \\
\hline Black & $149(18)$ \\
\hline Asian & $35(4)$ \\
\hline American Indian/Alaska Native & $17(2)$ \\
\hline Native Hawaiian/Pacific Islander & $7(1)$ \\
\hline Identified by $\geq 2$ races & $5(1)$ \\
\hline Unknown & $50(6)$ \\
\hline \multicolumn{2}{|l|}{ High-risk medical conditions $(n=794)^{b}$} \\
\hline No high-risk medical conditions & $341(43)$ \\
\hline One or more high-risk medical condition $c$ & $453(57)$ \\
\hline Pulmonary disease & $206(26)$ \\
\hline Asthma & $127(16)$ \\
\hline Neurologic disorder & $260(33)$ \\
\hline Neurodevelopmental disorder & $212(27)$ \\
\hline Cerebral palsy & $78(10)$ \\
\hline Neuromuscular disorder & $25(3)$ \\
\hline Seizure disorder & $126(16)$ \\
\hline Chromosome abnormality/genetic syndrome & $98(12)$ \\
\hline Congenital heart disease or other cardiac disease & $87(11)$ \\
\hline Immunosuppressive condition & $57(7)$ \\
\hline Cancer (received chemotherapy or radiation) & $31(4)$ \\
\hline Endocrine disorder & $45(6)$ \\
\hline Other conditions $^{d}$ & $30(4)$ \\
\hline \multicolumn{2}{|l|}{ Location of death $\left(n=823^{b}\right)$} \\
\hline Admitted to hospital & $537(65)$ \\
\hline Emergency department & $151(18)$ \\
\hline Outside hospital & $135(16)$ \\
\hline
\end{tabular}




\begin{tabular}{lc}
\hline Characteristic & Value \\
\hline Duration of illness, median $(\mathrm{IQR})\left(n=798^{b}\right), \mathrm{d}$ & $5(3-12)$ \\
$\leq 3$ days & $266(33)$ \\
$\leq 7$ days & $500(63)$ \\
One or more bacterial pathogen identified from normally sterile site $(n=387 b)$ & $154(40)$ \\
Staphylococcus aureus $\left(n=154^{\mathcal{e}}\right)$ & $76(49)$ \\
$\quad$ Methicillin-resistant $S$ aureus & $48(31)$ \\
$\quad$ Methicillin-sensitive $S$ aureus & $23(15)$ \\
$\quad$ Sensitivity testing not performed & $5(3)$ \\
Streptococcus pneumoniae & $22(14)$ \\
Complications during acute illness $\left(n=769^{b}\right)$ & \\
Pneumonia & $389(51)$ \\
Acute respiratory distress syndrome & $235(31)$ \\
Seizures & $83(11)$ \\
Encephalopathy/encephalitis & $68(9)$ \\
Viral coinfection & $39(5)$ \\
\hline
\end{tabular}

Data are presented as $n(\%)$ unless otherwise indicated; $N=830$. IQR, interquartile range.

a Hispanic or Latino ethnicity includes persons of any race. Data for other race categories do not include Hispanics.

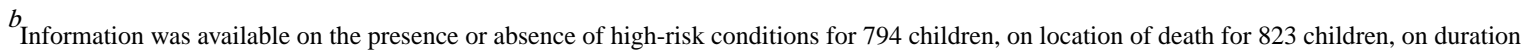
of illness for 798 children, on results of bacterial testing for 387 children who were tested, and on the presence or absence of complications for 769 children.

${ }^{c}$ More than 1 medical condition could be reported for each child.

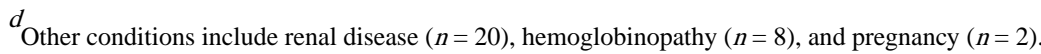

e Percentages reported out of 154 children with a bacterial coinfection. 
Wong et al.

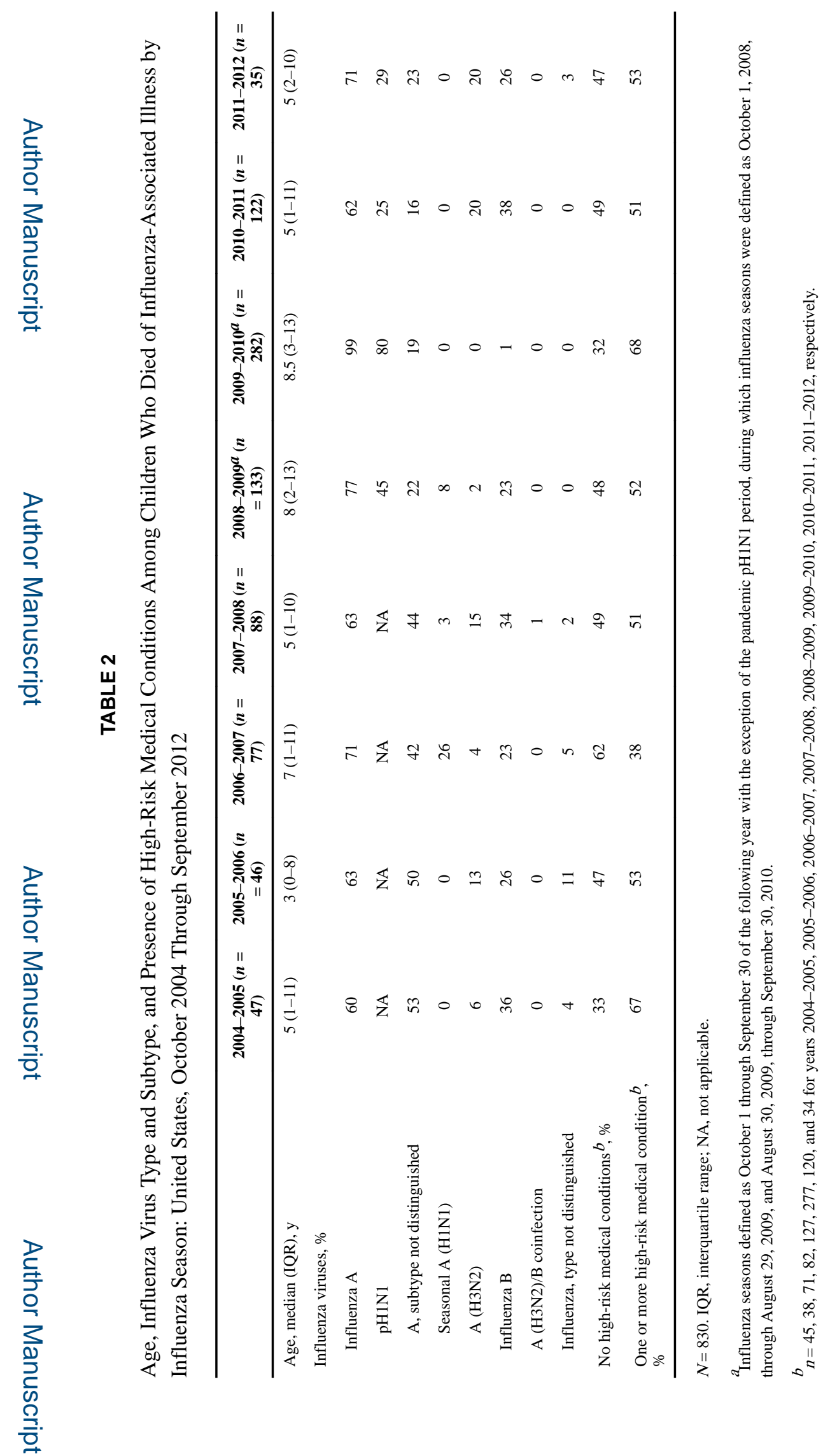

Pediatrics. Author manuscript; available in PMC 2018 July 02. 
TABLE 3

Selected Characteristics Among Children With Influenza-Associated Mortality by Presence of High-Risk Medical Conditions: United States, October 2004 Through September 2012

\begin{tabular}{|c|c|c|c|c|}
\hline Characteristic & $\begin{array}{l}\text { No High-Risk Medical } \\
\text { Conditions, }(n=341)\end{array}$ & $\begin{array}{l}\text { One or More High-Risk } \\
\text { Medical Condition, }(n=453)\end{array}$ & $\boldsymbol{P}$ & RR $(95 \%$ CI $)$ \\
\hline Age, median (IQR) ${ }^{a}, \mathrm{y}$ & $5(1-11)$ & $8(3-13)$ & $<.001$ & \\
\hline Age $<5$ years & $152 / 341(45)$ & $154 / 453(34)$ & & $1.3(1.1-1.6)$ \\
\hline Died before being admitted to hospital ${ }^{b}$ & $156 / 339(46)$ & $106 / 449(24)$ & & $1.9(1.6-2.4)$ \\
\hline Duration of illness $\leq 3$ days & $136 / 326(42)$ & $113 / 438(26)$ & & $1.6(1.3-2.0)$ \\
\hline $\begin{array}{l}\text { One or more bacterial pathogen identified from } \\
\text { normally sterile site }\end{array}$ & $86 / 155(55)$ & $63 / 222(28)$ & & $2.0(1.5-2.5)$ \\
\hline \multicolumn{5}{|l|}{ Type of complication } \\
\hline Pneumonia & $130 / 320(41)$ & $249 / 426(58)$ & & $0.7(0.6-0.8)$ \\
\hline Acute respiratory distress syndrome & $82 / 320(26)$ & $148 / 426(35)$ & & $0.7(0.6-0.9)$ \\
\hline Shock or sepsis & $104 / 320(33)$ & $97 / 426(23)$ & & $1.4(1.1-1.8)$ \\
\hline
\end{tabular}

Data are presented as $n(\%)$ unless otherwise indicated. IQR, interquartile range.

${ }^{a}$ Died in emergency department or outside of hospital. 